\title{
On a mixed Littlewood conjecture in Diophantine approximation
}

\author{
by \\ Yann Bugeaud (Strasbourg), Michael Drmota (Wien) \\ and Bernard de Mathan (Bordeaux)
}

1. Introduction. A famous open problem in simultaneous Diophantine approximation is the Littlewood conjecture [9]. It claims that, for every given pair $(\alpha, \beta)$ of real numbers, we have

$$
\liminf _{q \rightarrow \infty} q \cdot\|q \alpha\| \cdot\|q \beta\|=0
$$

where $\|\cdot\|$ denotes the distance to the nearest integer. The first significant contribution to this question goes back to Cassels and Swinnerton-Dyer [3] who showed that (1) holds when $\alpha$ and $\beta$ belong to the same cubic field. Further explicit examples of pairs $(\alpha, \beta)$ of real numbers satisfying (1) have been given in $[10,1]$. Despite some recent remarkable progress $[13,5]$ the Littlewood conjecture remains an open problem.

Recently, de Mathan and Teulié [12] proposed a "mixed Littlewood conjecture" that can be stated as follows. Let $\mathcal{D}=\left(d_{k}\right)_{k \in \mathbb{Z}}$ be a sequence of integers greater than or equal to 2 . Set $e_{0}=1$ and, for any $n \geq 1$,

$$
e_{n}=\prod_{0<k \leq n} d_{k} \text {. }
$$

For $q \in \mathbb{Z}$, set

$$
\begin{aligned}
w_{\mathcal{D}}(q) & =\sup \left\{n \in \mathbb{N}: q \in e_{n} \mathbb{Z}\right\}, \\
|q|_{\mathcal{D}} & =1 / e_{w_{\mathcal{D}}(q)}=\inf \left\{1 / e_{n}: q \in e_{n} \mathbb{Z}\right\} .
\end{aligned}
$$

When $\mathcal{D}$ is the constant sequence equal to $p$, where $p$ is a prime number, then $|\cdot|_{\mathcal{D}}$ is the usual $p$-adic absolute value $|\cdot|_{p}$, normalized by $|p|_{p}=p^{-1}$. In analogy with the Littlewood conjecture, de Mathan and Teulié asked whether

$$
\liminf _{q \rightarrow \infty} q \cdot\|q \alpha\| \cdot|q|_{\mathcal{D}}=0
$$

2000 Mathematics Subject Classification: 11J04, 11J61.

Research of Y. Bugeaud supported by the Austrian Science Fundation FWF, grant M822-N12. 
for every real number $\alpha$. They proved that (2) holds for every quadratic irrationality $\alpha$ when the sequence $\mathcal{D}$ is bounded.

In the present paper, we focus on the particular case when $\mathcal{D}$ is the constant sequence equal to a prime number. Thus, we investigate the following conjecture.

Mixed Littlewood Conjecture. For every real number $\alpha$ and every prime number $p$, we have

$$
\liminf _{q \rightarrow \infty} q \cdot\|q \alpha\| \cdot|q|_{p}=0 .
$$

Obviously, this holds if $\alpha$ is rational or has unbounded partial quotients. Thus, we only consider the case when $\alpha$ is an element of the set $\boldsymbol{B a d}$ of badly approximable numbers, where

$$
\boldsymbol{B a d}=\left\{\alpha \in \mathbb{R}: \inf _{q \geq 1} q \cdot\|q \alpha\|>0\right\} .
$$

We are concerned with the following question:

Problem 1. Is there any $\alpha$ in $\boldsymbol{B a d}$ which is irrational and not quadratic, and such that, for any prime number $p$, the pair $(\alpha, p)$ satisfies $(3)$ ?

As briefly outlined on p. 231 of [12], the answer to Problem 1 is positive when $\alpha$ lies in a subset of $\boldsymbol{B a d}$ with Hausdorff dimension 1; see also [6] for a stronger result. Nevertheless, these approaches do not provide any new explicit examples of pairs $(\alpha, p)$ satisfying (3) with $\alpha$ in $\boldsymbol{B a d}$. The purpose of the present note is precisely to construct explicitly uncountably many real numbers $\alpha$ in $\boldsymbol{B a d}$ such that the pair $(\alpha, p)$ satisfies (3) for any prime number $p$.

We further extend the problem posed by de Mathan and Teulié, by considering an "inhomogeneous mixed Littlewood conjecture". We ask whether

$$
\liminf _{q \rightarrow \infty} q \cdot\|q \alpha\| \cdot|q-y|_{p}=0
$$

for any real number $\alpha$, any prime number $p$ and any $y$ in $\mathbb{Z}_{p}$, the ring of $p$-adic integers. It turns out that our methods allow us to establish (4) for a wide class of real numbers $\alpha$ in $\boldsymbol{B a d}$.

Our proofs heavily depend on $p$-adic analysis, and our key tool is the $p$-adic logarithm function. It is not clear to us whether our Theorem 1 has a real analogue, or an analogue for formal power series over a finite field.

2. Results. Our main result shows that (3) holds for any real number $\alpha$ whose sequence of partial quotients is, in some sense, quasi-periodic.

Theorem 1. Let $\alpha$ be in Bad and write

$$
\alpha=\left[a_{0} ; a_{1}, a_{2}, \ldots\right] .
$$


Let $T \geq 1$ be an integer and $b_{1}, \ldots, b_{T}$ be positive integers. If there exist two sequences $\left(m_{k}\right)_{k \geq 1}$ and $\left(h_{k}\right)_{k \geq 1}$ of positive integers with $\left(h_{k}\right)_{k \geq 1}$ being unbounded and

$$
a_{m_{k}+j+n T}=b_{j} \quad \text { for every } j=1, \ldots, T \text { and every } n=0, \ldots, h_{k}-1,
$$
then

$$
\liminf _{q \rightarrow \infty} q \cdot\|q \alpha\| \cdot|q-y|_{p}=0
$$

for every prime number $p$ and every $y \in \mathbb{Z}_{p}$. If , furthermore, there exists a constant $C$ such that

$$
m_{k} \leq C h_{k} \quad \text { for } k \geq 1
$$

then

$$
\liminf _{q \rightarrow \infty} q \cdot \log q \cdot\|q \alpha\| \cdot|q-y|_{p}<\infty,
$$

for every prime number $p$ and every $y \in \mathbb{Z}_{p}$.

It is worth rephrasing the assumption of Theorem 1 by using the terminology of combinatorics on words. Let $\alpha=\left[a_{0} ; a_{1}, a_{2}, \ldots\right]$ be in $\boldsymbol{B a d}$ and view its sequence of partial quotients as the infinite word $\mathbf{a}=a_{0} a_{1} a_{2} \ldots$ on the alphabet $\mathcal{A}=\{1, \ldots, M\}$, where $M$ is an upper bound for the $a_{i}$ 's. Theorem 1 asserts that if there exists a finite, non-empty word $B$ on the alphabet $\mathcal{A}$ such that, for every $k \geq 1$, the concatenation of $k$ copies of $B$ occurs in the word $\mathbf{a}$, then (5) holds for every prime number $p$ and every $y$ in $\mathbb{Z}_{p}$. Consequently, Theorem 1 provides an uncountable, explicit class of badly approximable real numbers for which the mixed Littlewood conjecture, and even the inhomogeneous mixed Littlewood conjecture, is true.

We further mention that if the real number $\alpha$ satisfies the assumption of Theorem 1 with sequences $\left(m_{k}\right)_{k \geq 1}$ and $\left(h_{k}\right)_{k \geq 1}$ such that (6) holds, then $\alpha$ is either a quadratic irrationality, or a transcendental number. This follows from Theorem 3.2 of [2].

We display an immediate consequence of the proof of Theorem 1.

THEOREM 2. Let $\alpha$ be a quadratic real number. Let $p$ be a prime number. For any $y$ in $\mathbb{Z}_{p}$, there exist a positive constant $c(\alpha, p, y)$, depending only on $\alpha, p$ and $y$, and arbitrarily large positive integers $q$ with

$$
\|q \alpha\| \leq c(\alpha, p, y) / q
$$

and

$$
|q-y|_{p} \leq c(\alpha, p, y) / \log q .
$$

In particular, (7) holds.

The case $y=0$ was already established in Théorème 2.1 of [12]. Notice that the latter result actually covers the more general case of a bounded 
sequence $\mathcal{D}$ of positive integers. In view of [11], we cannot replace $\log q$ in (8) by $(\log q)^{\lambda}$ with $\lambda$ very large.

Theorem 2 also holds for an arbitrary bounded sequence $\mathcal{D}$ when $y$ is an integer.

Using some of the ideas occurring in the proof of Theorem 1, we get another uncountable, explicit class of badly approximable real numbers for which the mixed Littlewood conjecture, and even the inhomogeneous mixed Littlewood conjecture, is true.

TheOREM 3. Let $\alpha=\left[a_{0} ; a_{1}, a_{2}, \ldots\right]$ be in Bad. If for every integer $h \geq 1$ there exists an integer $T$ such that

$$
a_{j+n T}=a_{j} \quad \text { for every } j=1, \ldots, T \text { and every } n=0, \ldots, h,
$$

then, for any prime number $p$ and any integer $y$,

$$
\liminf _{q \rightarrow \infty} q \cdot\|q \alpha\| \cdot|q-y|_{p}<\infty .
$$

More generally, Theorem 3 holds for an arbitrary (bounded) sequence $\mathcal{D}$. Its analogue in the function field case can also be easily established.

Using arguments from [13], we deduce that (4) holds for "many" real numbers $\alpha$ with bounded partial quotients.

THEOREM 4. The set of real numbers $\alpha$ with bounded partial quotients for which

$$
\liminf _{q \rightarrow \infty} q \cdot\|q \alpha\| \cdot|q-y|_{p}=0
$$

for every prime number $p$ and every $y$ in $\mathbb{Z}$ has Hausdorff dimension 1.

A stronger result holds when $y=0$. Namely, Einsiedler and Kleinbock [6] established that the set of $\alpha$ for which (3) does not hold has Hausdorff dimension 0. Presumably, their approach could be modified to get an analogous result for (4), but this is not clear to us.

In view of Theorem 1, we would like to address the following problem.

Problem 2. Let $\varepsilon$ be a positive real number. Find a real number $\alpha$ in $\boldsymbol{B a d}$, a prime number $p$, and a rational integer $y$ such that

$$
\liminf _{q \rightarrow \infty} q^{1+\varepsilon} \cdot\|q \alpha\| \cdot|q-y|_{p}<\infty
$$

It follows from the $p$-adic analogue of the Schmidt subspace theorem, established by Schlickewei [15], that any real number $\alpha$ satisfying (9) must be transcendental. Apparently, our construction does not allow us to tackle Problem 2. Nevertheless, by using the folding lemma recalled in Section 6, it is possible to give a positive answer to Problem 2. 
THEOREM 5. For any prime number $p$, there exists a real number $\alpha$ in Bad such that

$$
\liminf _{q \rightarrow \infty} q^{2} \cdot\|q \alpha\| \cdot|q|_{p} \leq 1 .
$$

Since (9) cannot hold with $\varepsilon>1$ and $\alpha$ in $\boldsymbol{B a d}$, Theorem 5 is best possible.

We end this section with a third question.

Problem 3. Given $\alpha$ in $\boldsymbol{B a d}$, is there a prime number $p$ such that (3) holds for the pair $(\alpha, p)$ ?

Apparently, there is no contribution towards Problem 3, which seems to be quite difficult.

\section{Proof of Theorem 1}

Preliminaries to the proof. Let $\left(q_{n}\right)_{n \geq-1}$ be the sequence of the denominators of the convergents of $\alpha$. These numbers satisfy the recurrence relation

$$
q_{n}=a_{n} q_{n-1}+q_{n-2}
$$

with $q_{0}=1$ and $q_{-1}=0$. For $n \geq 0$, set

$$
Q_{n}=\left(\begin{array}{c}
q_{n} \\
q_{n-1}
\end{array}\right)
$$

We can write

$$
Q_{n}=P_{n} Q_{n-1} \quad \text { where } \quad P_{n}=\left(\begin{array}{cc}
a_{n} & 1 \\
1 & 0
\end{array}\right)
$$

Set

$$
M_{j}=\left(\begin{array}{cc}
b_{j} & 1 \\
1 & 0
\end{array}\right), \quad j=1, \ldots, T, \quad M=M_{T} \ldots M_{1} .
$$

Replacing if necessary $b_{1}, \ldots, b_{T}$ by $b_{1}, \ldots, b_{T}, b_{1}, \ldots, b_{T}$, we may replace $T$ by $2 T$, and thus we can suppose $\operatorname{det} M=1$. The matrix $M$ is diagonalizable and its eigenvalues are quadratic units $\omega>1$ and $1 / \omega$. We have $N_{\mathbb{Q}(\omega) / \mathbb{Q}} \omega$ $=1$. Set $a=\operatorname{Tr}_{\mathbb{Q}(\omega) / \mathbb{Q}} \omega$. Then $a>2$ is a positive integer, and $\omega$ is a root of the polynomial $X^{2}-a X+1$. We have $Q_{m_{k}+n T}=M Q_{m_{k}+(n-1) T}$ for every $1 \leq n \leq h_{k}$, hence $Q_{m_{k}+n T}=M^{n} Q_{m_{k}}$. The Cayley-Hamilton theorem implies that, for $2 \leq n \leq h_{k}$, we have

$$
\begin{aligned}
Q_{m_{k}+n T} & =M^{2} Q_{m_{k}+(n-2) T}=(a M-I) Q_{m_{k}+(n-2) T} \\
& =a Q_{m_{k}+(n-1) T}-Q_{m_{k}+(n-2) T} .
\end{aligned}
$$

Hence the sequence $\left(q_{m_{k}+n T}\right)_{n \geq 0}$ satisfies

$$
q_{m_{k}+n T}=a q_{m_{k}+(n-1) T}-q_{m_{k}+(n-2) T}
$$

when $2 \leq n \leq h_{k}$. 
Note that $Q_{m_{k}+1+n T}=M^{\prime} Q_{m_{k}+1+(n-1) T}$ for $0<n<h_{k}$, where $M^{\prime}=$ $M_{1} M_{T} \ldots M_{2}=M_{1} M M_{1}^{-1}$. Hence, $M^{\prime}$ has the same characteristic polynomial as $M$, and the sequence $\left(q_{m_{k}+1+n T}\right)_{n \geq 0}$ also satisfies the recurrence

$$
q_{m_{k}+1+n T}=a q_{m_{k}+1+(n-1) T}-q_{m_{k}+1+(n-2) T}
$$

when $2 \leq n<h_{k}$. Consequently, we may replace $m_{k}$ by $m_{k}+1$. Since $q_{m_{k}}$ and $q_{m_{k}+1}$ are coprime, we can, without any loss of generality, suppose that $p$ does not divide $q_{m_{k}}$.

We have

$$
q_{m_{k}+n T}=A_{k} \omega^{n}+B_{k} \omega^{-n}
$$

for $0 \leq n \leq h_{k}$, where $A_{k}$ and $B_{k}$ are given by (11) with $n=0$ and $n=1$ :

$$
\begin{aligned}
& A_{k}=\frac{\omega q_{m_{k}+T}-q_{m_{k}}}{\omega^{2}-1}, \\
& B_{k}=-\omega \frac{q_{m_{k}+T}-\omega q_{m_{k}}}{\omega^{2}-1} .
\end{aligned}
$$

We also denote by $\omega$ a zero of the polynomial $X^{2}-a X+1$ in the algebraic closure of $\mathbb{Q}_{p}$. We still denote by $|\cdot|_{p}$ the $p$-adic value extended to this field. As $\omega$ is a unit, we have $|\omega|_{p}=1$. The formulæ (12) and (13) hold in $\mathbb{Q}_{p}$. Since $\omega$ and $\omega^{-1}$ are conjugate in $\mathbb{Q}(\omega)$, and so are $A_{k}$ and $B_{k}$, we can change $A_{k}$ into $B_{k}$ by changing the embedding of $\omega$ in $\mathbb{Q}_{p}(\omega)$-it is convenient to make this embedding depend upon $k$. We can thus suppose that $\left|A_{k}\right|_{p} \geq\left|B_{k}\right|_{p}$ (i.e., $\left|q_{m_{k}+T}-\omega q_{m_{k}}\right|_{p} \leq\left|\omega q_{m_{k}+T}-q_{m_{k}}\right|_{p}$ ). Since we suppose that $\left|A_{k}+B_{k}\right|_{p}=\left|q_{m_{k}}\right|_{p}=1$, we then have $1 \leq\left|A_{k}\right|_{p} \leq 1 /\left|\omega^{2}-1\right|_{p}$.

The field $\mathbb{Q}_{p}(\omega)$ is complete. The ball

$$
G=\left\{x \in \mathbb{Q}_{p}(\omega):|x-1|_{p}<p^{-1 /(p-1)}\right\}
$$

is a subgroup of finite index in the multiplicative group $\left\{x \in \mathbb{Q}_{p}(\omega):|x|_{p}=1\right\}$. Hence, replacing again $T$ by $\ell T$, therefore $\omega$ by $\omega^{\ell}$, where $\ell$ is a suitable positive integer, we may also suppose that $|\omega-1|_{p}<p^{-1 /(p-1)}$.

In what follows, we shall make use of the $p$-adic logarithm function, which is defined on the multiplicative group $\left\{x \in \mathbb{Q}_{p}(\omega):|x-1|_{p}<1\right\}$ in $\mathbb{Q}_{p}(\omega)$ by

$$
\log x=\sum_{n=1}^{\infty}(-1)^{n-1}(x-1)^{n} / n .
$$

We have

$$
\log x y=\log x+\log y \quad \text { for } x, y \text { in }\left\{x \in \mathbb{Q}_{p}(\omega):|x-1|_{p}<1\right\},
$$

and, for every $x, y$ in $G$,

$$
|\log x|_{p}=|x-1|_{p}
$$


and

$$
|\log x-\log y|_{p}=\left|\log \frac{x}{y}\right|_{p}=\left|\frac{x}{y}-1\right|_{p}=|x-y|_{p} .
$$

The constants implied in the symbols $\ll$ and $\asymp$ occurring below will only depend upon $b_{1}, \ldots, b_{T}$ and on $p$.

The proofs in the cases $y=0$ and $y \neq 0$ are rather different. First, we deal with the case $y=0$, which is slightly more difficult.

An auxiliary result for the case $y=0$. Keep the above notations and set either

$$
\varepsilon_{k}=\left|\omega^{2}-1\right|_{p} \cdot\left|q_{m_{k}+T}-\omega q_{m_{k}}\right|_{p}
$$

or

$$
\varepsilon_{k}=p^{-1 / 2} \cdot\left|\omega^{2}-1\right|_{p} \cdot\left|q_{m_{k}+T}-\omega q_{m_{k}}\right|_{p},
$$

in such a way that $\varepsilon_{k}<1$ is an integral power of $p$.

LEMMA 1. There exist integers $x_{k}$ and $y_{k}$, with $\left|y_{k}\right|_{p}=1$, such that

$$
\begin{aligned}
\left|x_{k} q_{m_{k}}+y_{k} q_{m_{k}+T}\right|_{p} & \leq \varepsilon_{k}, \\
\max \left\{\left|x_{k}\right|,\left|y_{k}\right|\right\} & \ll \varepsilon_{k}^{-1 / 2} .
\end{aligned}
$$

Furthermore,

$$
\left|x_{k} q_{m_{k}}+y_{k} q_{m_{k}+T}\right|_{p} \leq\left|\omega^{2}-1\right|_{p}\left|x_{k}+y_{k} \omega\right|_{p} \ll\left(\max \left\{\left|x_{k}\right|,\left|y_{k}\right|\right\}\right)^{-2} .
$$

To prove Lemma 1, we need the following version of Liouville's lemma.

Lemma 2. For any integers $X$ and $Y$, not both zero,

$$
|X+Y \omega|_{p} \gg(\max \{|X|,|Y|\})^{-2} \text {. }
$$

Proof. We have

$$
\left|N_{\mathbb{Q}(\omega) / \mathbb{Q}}(X+Y \omega)\right| \ll \max \{|X|,|Y|\}^{2}
$$

and

$$
\left|N_{\mathbb{Q}(\omega) / \mathbb{Q}}(X+Y \omega)\right|_{p}=|(X+Y \omega)(X+Y / \omega)|_{p} \leq|X+Y \omega|_{p} .
$$

As $N_{\mathbb{Q}(\omega) / \mathbb{Q}}(X+Y \omega)$ is a non-zero integer, we get

$$
\left|N_{\mathbb{Q}(\omega) / \mathbb{Q}}(X+Y \omega)\right| \cdot\left|N_{\mathbb{Q}(\omega) / \mathbb{Q}}(X+Y \omega)\right|_{p} \geq 1
$$

It then follows that $|X+Y \omega|_{p} \max \{|X|,|Y|\}^{2} \gg 1$, as claimed.

Proof of Lemma 1. Let $s$ be a positive integer. By the pigeonhole principle, there exist integers $x_{k, s}$ and $y_{k, s}$, not both zero, such that

$$
\begin{aligned}
\left|x_{k, s} q_{m_{k}}+y_{k, s} q_{m_{k}+T}\right|_{p} & \leq \varepsilon_{k} p^{-s}, \\
\max \left\{\left|x_{k, s}\right|,\left|y_{k, s}\right|\right\} & \leq \varepsilon_{k}^{-1 / 2} p^{s / 2} .
\end{aligned}
$$

First, we prove that there exists a non-negative integer $S$, depending only on $\omega$, such that if $s>S$, then $y_{k, s}$ cannot be divisible by $p^{s}$. Indeed, let 
$\sigma$ be a positive integer with $0 \leq \sigma \leq s$ such that $y_{k, s}$ is divisible by $p^{\sigma}$. Then $x_{k, s}$ as well is divisible by $p^{\sigma}$. Indeed, we have $\left|y_{k, s} q_{m_{k}+T}\right|_{p} \leq p^{-\sigma}$ and $\left|x_{k, s} q_{m_{k}}+y_{k, s} q_{m_{k}+T}\right|_{p} \leq p^{-\sigma}$, hence $\left|x_{k, s} q_{m_{k}}\right|_{p} \leq p^{-\sigma}$. Since we have assumed that $\left|q_{m_{k}}\right|_{p}=1$, we get $\left|x_{k, s}\right|_{p} \leq p^{-\sigma}$. Setting $x_{k, s}^{\prime}=p^{-\sigma} x_{k, s}$ and $y_{k, s}^{\prime}=p^{-\sigma} y_{k, s}$, we deduce from (17) that

$$
\left|x_{k, s}^{\prime}+y_{k, s}^{\prime} \frac{q_{m_{k}+T}}{q_{m_{k}}}\right|_{p} \leq \varepsilon_{k} p^{-s+\sigma}
$$

and from (18) that

$$
\max \left\{\left|x_{k, s}^{\prime}\right|,\left|y_{k, s}^{\prime}\right|\right\} \leq \varepsilon_{k}^{-1 / 2} p^{s / 2-\sigma} .
$$

Hence, writing

$$
x_{k, s}^{\prime}+y_{k, s}^{\prime} \omega=x_{k, s}^{\prime}+y_{k, s}^{\prime} \frac{q_{m_{k}+T}}{q_{m_{k}}}+y_{k, s}^{\prime}\left(\omega-\frac{q_{m_{k}+T}}{q_{m_{k}}}\right),
$$

and noticing that (19) implies that

$$
\left|x_{k, s}^{\prime}+y_{k, s}^{\prime} \frac{q_{m_{k}+T}}{q_{m_{k}}}\right|_{p}<\left|q_{m_{k}+T}-\omega q_{m_{k}}\right|_{p},
$$

we get

$$
\left|x_{k, s}^{\prime}+y_{k, s}^{\prime} \omega\right|_{p} \leq\left|q_{m_{k}+T}-\omega q_{m_{k}}\right|_{p} \ll \varepsilon_{k} .
$$

Then by (20),

$$
\left|x_{k, s}^{\prime}+y_{k, s}^{\prime} \omega\right|_{p} \max \left\{\left|x_{k, s}^{\prime}\right|,\left|y_{k, s}^{\prime}\right|\right\}^{2} \ll p^{s-2 \sigma} .
$$

If $\sigma=s$, we thus get

$$
\left|x_{k, s}^{\prime}+y_{k, s}^{\prime} \omega\right|_{p} \max \left\{\left|x_{k, s}^{\prime}\right|,\left|y_{k, s}^{\prime}\right|\right\}^{2} \leq p^{-s},
$$

and it follows from Lemma 2 that we must have $s \ll 1$, i.e., $s \leq S$, where $S \geq 0$ is an integral constant, depending only upon $\omega$. Thus, if $s>S$, then $y_{k, s}$ cannot be divisible by $p^{s}$. Take $s=S+1$. If we define $\sigma$ by $\left|y_{k, s}\right|_{p}=p^{-\sigma}$, then $\sigma<s$. Set $x_{k}=p^{-\sigma} x_{k, s}$ and $y_{k}=p^{-\sigma} y_{k, s}$, so that

$$
\left|y_{k}\right|_{p}=1 \text {. }
$$

By (19) and (20), as $s=S+1$, we deduce (14) and (15). To conclude, let us show (16). Indeed, as $\varepsilon_{k}<\left|q_{m_{k}+T}-\omega q_{m_{k}}\right|_{p}$, we deduce from (22), (14) and (21) that

$$
\left|x_{k}+y_{k} \omega\right|_{p}=\left|q_{m_{k}+T}-\omega q_{m_{k}}\right|_{p} .
$$

Accordingly, (14) and (15) lead to the desired conclusion.

Completion of the proof for the case $y=0$. For any $k \geq 1$, let $x_{k}$ and $y_{k}$ be the integers given by Lemma 1 . For any $n \geq 0$, set

$$
Q_{k, n}=x_{k} q_{m_{k}+n T}+y_{k} q_{m_{k}+(n+1) T} .
$$


Our aim is to prove that there exists an integer $n(k) \leq h_{k}-1$ such that $Q_{k, n(k)} \neq 0$, and

$$
\inf _{k \geq 1}\left|Q_{k, n(k)}\right| \cdot\left|Q_{k, n(k)}\right|_{p} \cdot\left\|Q_{k, n(k)} \alpha\right\|=0 .
$$

First, we note that, for every $0 \leq n<h_{k}$, we have

$$
\begin{aligned}
\left|Q_{k, n}\right| & \ll \max \left\{\left|x_{k}\right|,\left|y_{k}\right|\right\} q_{m_{k}+n T}, \\
\left\|Q_{k, n} \alpha\right\| & \ll \max \left(\left|x_{k}\right|,\left|y_{k}\right|\right) / q_{m_{k}+n T} .
\end{aligned}
$$

We thus get

$$
\left|Q_{k, n}\right| \cdot\left\|Q_{k, n} \alpha\right\| \ll \max \left\{\left|x_{k}\right|,\left|y_{k}\right|\right\}^{2} .
$$

Further, with $A_{k}$ and $B_{k}$ being defined as in (12) and (13), we can write

$$
Q_{k, n}=C_{k} \omega^{n}+D_{k} \omega^{-n}
$$

where

$$
C_{k}=A_{k}\left(x_{k}+y_{k} \omega\right), \quad D_{k}=B_{k}\left(x_{k}+y_{k} \omega^{-1}\right) .
$$

Since $\left|A_{k}\right|_{p} \geq 1$, we deduce from Lemma 1 that

$\left|C_{k}+D_{k}\right|_{p}=\left|x_{k} q_{m_{k}}+y_{k} q_{m_{k}+T}\right|_{p} \leq\left|\omega^{2}-1\right|_{p} \cdot\left|x_{k}+y_{k} \omega\right|_{p} \leq\left|\omega^{2}-1\right|_{p} \cdot\left|C_{k}\right|_{p}$.

Hence, $\left|C_{k}+D_{k}\right|_{p}<\left|C_{k}\right|_{p}$, and thus $\left|C_{k}\right|_{p}=\left|D_{k}\right|_{p}$. We then get

$$
\left|1+D_{k} / C_{k}\right|_{p} \leq\left|\omega^{2}-1\right|_{p}<p^{-1 /(p-1)} .
$$

Write

$$
Q_{k, n}=C_{k} \omega^{-n}\left(D_{k} / C_{k}+\omega^{2 n}\right) .
$$

Since $\left|C_{k}\right|_{p} \ll\left|x_{k}+y_{k} \omega\right|_{p} \ll\left(\max \left\{\left|x_{k}\right|,\left|y_{k}\right|\right\}\right)^{-2}$, we thus get

$$
\left|Q_{k, n}\right|_{p} \ll\left(\max \left\{\left|x_{k}\right|,\left|y_{k}\right|\right\}\right)^{-2}\left|D_{k} / C_{k}+\omega^{2 n}\right|_{p}
$$

and, by (24),

$$
\left|Q_{k, n}\right| \cdot\left|Q_{k, n}\right|_{p} \cdot\left\|Q_{k, n} \alpha\right\| \ll\left|D_{k} / C_{k}+\omega^{2 n}\right|_{p}
$$

Now, inequality (25) enables us to use the $p$-adic logarithm in the domain $G$ where $|\log x-\log y|_{p}=|x-y|_{p}$. As

$$
\left|D_{k} / C_{k}+\omega^{2 n}\right|_{p}=\left|\log \left(-D_{k} / C_{k}\right)-2 n \log \omega\right|_{p},
$$

we may also write (26) as

$$
\left|Q_{k, n}\right| \cdot\left|Q_{k, n}\right|_{p} \cdot\left\|Q_{k, n} \alpha\right\| \ll\left|\frac{\log \left(-D_{k} / C_{k}\right)}{2 \log \omega}-n\right|_{p} .
$$

Now let us show that $\left(\log \left(-D_{k} / C_{k}\right)\right) /(2 \log \omega)$ lies in $\mathbb{Q}_{p}$. This is trivial if $\omega \in \mathbb{Q}_{p}$. In the case where $\omega \notin \mathbb{Q}_{p}$, there exists a unique $\mathbb{Q}_{p}$-automorphism $\sigma$ of $\mathbb{Q}_{p}(\omega)$, different from the identity. We have $\sigma(\omega)=1 / \omega$, and $\sigma$ is isometrical. We have $\sigma(\log \omega)=\log (\sigma(\omega))=-\log \omega$, and $\sigma\left(-D_{k} / C_{k}\right)=$ $-C_{k} / D_{k}$, since $\sigma\left(C_{k}\right)=D_{k}$. We thus have

$$
\sigma\left(\log \left(-D_{k} / C_{k}\right)\right)=\log \left(-C_{k} / D_{k}\right)=-\log \left(-D_{k} / C_{k}\right) .
$$


Hence,

$$
\sigma\left(\frac{\log \left(-D_{k} / C_{k}\right)}{2 \log \omega}\right)=\frac{\log \left(-D_{k} / C_{k}\right)}{2 \log \omega}
$$

and so the element $\log \left(-D_{k} / C_{k}\right) /(2 \log \omega)$ of $\mathbb{Q}_{p}(\omega)$ lies in $\mathbb{Q}_{p}$. Further, it lies in $\mathbb{Z}_{p}$, since

$$
\left|\log \left(-D_{k} / C_{k}\right)\right|_{p}=\left|D_{k} / C_{k}+1\right|_{p} \leq\left|\omega^{2}-1\right|_{p}=|2 \log \omega|_{p},
$$

by (25). If $t_{k}$ is a positive integer with

$$
\frac{1}{2 p} h_{k}<p^{t_{k}} \leq \frac{1}{2} h_{k},
$$

then there exists an integer $n$, with $0 \leq n<p^{t_{k}}$, such that

$$
\left|\frac{\log \left(-D_{k} / C_{k}\right)}{2 \log \omega}-n\right|_{p} \leq p^{-t_{k}}
$$

Replacing if necessary $n$ by $n+p^{t_{k}}$, we may ensure that $\log \left(-D_{k} / C_{k}\right) \neq$ $2 n \log \omega$, hence $D_{k} / C_{k}+\omega^{2 n} \neq 0$. Taking either $n(k)=n$ or $n(k)=n+p^{t_{k}}$, we have thus constructed an integer $n(k)$ satisfying $0 \leq n(k)<h_{k}, Q_{k, n(k)}$ $\neq 0$ and

$$
\left|Q_{k, n(k)}\right| \cdot\left|Q_{k, n(k)}\right|_{p} \cdot\left\|Q_{k, n(k)} \alpha\right\| \ll p^{-t_{k}} \ll \frac{1}{h_{k}} .
$$

Since the sequence $\left(h_{k}\right)_{k \geq 1}$ is unbounded, we have

$$
\inf _{k \geq 1}\left|Q_{k, n(k)}\right| \cdot\left|Q_{k, n(k)}\right|_{p} \cdot\left\|Q_{k, n(k)} \alpha\right\|=0 .
$$

It remains for us to prove that the estimate (7) holds when (6) is satisfied. Note that as the partial quotients of $\alpha$ are bounded, by (23) we have

$$
\log \left|Q_{k, n(k)}\right| \ll \log \max \left\{\left|x_{k}\right|,\left|y_{k}\right|\right\}+m_{k}+n(k),
$$

hence

$$
\log \left|Q_{k, n(k)}\right| \ll \log \max \left\{\left|x_{k}\right|,\left|y_{k}\right|\right\}+m_{k}+h_{k} .
$$

Now, by (15) and Lemma 2, we have

$$
\max \left\{\left|x_{k}\right|,\left|y_{k}\right|\right\} \ll\left|q_{m_{k}+T}-\omega q_{m_{k}}\right|_{p}^{-1 / 2} \ll q_{m_{k}} .
$$

Thus, $\log \max \left\{\left|x_{k}\right|,\left|y_{k}\right|\right\} \ll m_{k}$ and so

$$
\log \left|Q_{k, n(k)}\right| \ll m_{k}+h_{k} .
$$

Accordingly, if (6) is satisfied, then $\log \left|Q_{k, n(k)}\right| \ll h_{k}$, and (7) follows from (27).

The case $y \neq 0$. We shall prove that for any non-zero $y$ in $\mathbb{Z}_{p}$, there exists an infinite set $\mathcal{Q}$ of positive integers $Q$ satisfying

$$
Q \cdot\|Q \alpha\| \ll 1
$$


and

$$
\liminf _{\mathcal{Q} \ni Q \rightarrow \infty}|Q-y|_{p}=0 .
$$

Note that if (29) is true for $y$, then it is true for $M y$, where $M$ is any positive integer. Indeed, we may replace $Q$ by $M Q$, while preserving (28). Thus it is enough to prove (29) when $|y|_{p}=1$. Actually we shall construct a set of positive integers $Q$, with (28), such that the $p$-adic topological closure of this set contains the unit circumference $|y-1|_{p}=1$. For this purpose, it is enough to construct a set of $Q$, with (28), whose $p$-adic closure contains the ball $|y-1|_{p} \leq p^{-\lambda}$ for some positive integer $\lambda$. Indeed, if we replace the set of $Q$ by the set of $\mu Q$, where $\mu$ runs through the integers $0<\mu<p^{\lambda}$ with $|\mu|_{p}=1$, the $p$-adic closure of this last set will contain the unit circumference $|y-1|_{p}=1$.

First, suppose that $p \neq 2$. Let $\left(m_{k}\right)_{k \geq 1}$ and $\left(h_{k}\right)_{k \geq 1}$ be as in the statement of the theorem. Define $A_{k}$ and $B_{k}$ by (12) and (13). Recall that we suppose that $\left|q_{m_{k}}\right|_{p}=1$ and $\left|A_{k}\right|_{p} \geq\left|B_{k}\right|_{p}$, hence $\left|A_{k}\right|_{p} \geq 1$. We may also suppose that $\left|A_{k}-B_{k}\right|_{p} \geq|\omega-1|_{p}$. Indeed, we may replace $q_{m_{k}}$ by $q_{m_{k}+T}$ or $q_{m_{k}+2 T}$, preserving the condition $\left|q_{m_{k}}\right|_{p}=1$, since (10) ensures that if $q_{m_{k}}$ is not divisible by $p$, then neither is $q_{m_{k}+T}$ or $q_{m_{k}+2 T}$. Now, if we replace $q_{m_{k}}$ by $q_{m_{k}+T}$ (resp. $q_{m_{k}+2 T}$ ), then $A_{k}$ is replaced by $A_{k} \omega\left(\operatorname{resp} . A_{k} \omega^{2}\right)$, and $B_{k}$ is replaced by $B_{k} \omega^{-1}$ (resp. $B_{k} \omega^{-2}$ ). If $\left|A_{k}-B_{k}\right|_{p}<|\omega-1|_{p}$, then

$$
\begin{aligned}
\left|A_{k} \omega-B_{k} \omega^{-1}\right|_{p} & =\left|A_{k}\left(\omega-\omega^{-1}\right)+\left(A_{k}-B_{k}\right) \omega^{-1}\right|_{p}=\left|A_{k}\left(\omega-\omega^{-1}\right)\right|_{p} \\
& \geq\left|\omega^{2}-1\right|_{p}=|\omega-1|_{p}
\end{aligned}
$$

in view of the properties of the logarithm function, since $|\omega-1|_{p}<p^{-1 /(p-1)}$. In the same way, if $\left|A_{k}-B_{k}\right|_{p}<|\omega-1|_{p}$, then $\left|A_{k} \omega^{2}-B_{k} \omega^{-2}\right|_{p} \geq|\omega-1|_{p}$. Accordingly, by these changes, we may suppose that $\left|q_{m_{k}}\right|_{p}=1$ and

$$
\left|A_{k}-B_{k}\right|_{p} \geq|\omega-1|_{p}
$$

Let $\lambda$ be the positive integer such that

$$
|\omega-1|_{p}^{2}=p^{-\lambda+1} \text {. }
$$

As $\left|q_{m_{k}}\right|_{p}=1$ there exists an integer $L_{k}$ with $0<L_{k}<p^{\lambda}$ and $\left|L_{k}\right|_{p}=1$ such that

$$
L_{k}\left(A_{k}+B_{k}\right) \equiv 1 \bmod p^{\lambda}
$$

We shall prove that the $p$-adic closure of the set composed by the integers $L_{k} q_{m_{k}+n T}$ for $k \geq 1$ and $0 \leq n \leq h_{k}$ contains the ball $|y-1|_{p} \leq p^{-\lambda}$. Note that, $L_{k}$ being bounded, the integers $L_{k} q_{m_{k}+n T}$ satisfy (28), and thus the result will be proved.

Set $L_{k} A_{k}=A_{k}^{\prime}$ and $L_{k} B_{k}=B_{k}^{\prime}$. For $0 \leq n \leq h_{k}$, we have

$$
L_{k} q_{m_{k}+n T}=A_{k}^{\prime} \omega^{n}+B_{k}^{\prime} \omega^{-n} \text {. }
$$


Consider the map $\varphi_{k}$ from the ball $\left\{x:|x-1|_{p}<1\right\}$ of $\mathbb{Q}_{p}(\omega)$ into $\mathbb{Q}_{p}(\omega)$ such that

$$
\varphi_{k}(x)=A_{k}^{\prime} x+B_{k}^{\prime} x^{-1} .
$$

Let $y \in \mathbb{Q}_{p}$ with

$$
|y-1|_{p} \leq p^{-\lambda}
$$

We shall find $x \in \mathbb{Q}_{p}(\omega)$ such that $\varphi_{k}(x)=y$. We must have $A_{k}^{\prime} x^{2}-y x+B_{k}^{\prime}$ $=0$. Accordingly we take

$$
x=\frac{y+\sqrt{y^{2}-4 A_{k}^{\prime} B_{k}^{\prime}}}{2 A_{k}^{\prime}} .
$$

However, we must make precise the meaning of the symbol $\sqrt{ } \cdot$. We define the function $\sqrt{\cdot}$ over the ball $\left\{z:|z-1|_{p} \leq p^{-1}\right\}$ in $\mathbb{Q}_{p}$, with values in the same ball, by the equality $\log \sqrt{z}=\frac{1}{2} \log z$ (that is to say, $\sqrt{z}=\exp \frac{\log z}{2}$, where $\exp u=\sum_{n=0}^{\infty} u^{n} / n$ ! for $\left.|u|_{p} \leq p^{-1}\right)$. Therefore we have

$$
|\sqrt{z}-1|_{p}=|z-1|_{p}
$$

Now, by (31) and (32), we have

$$
\left|y^{2}-4 A_{k}^{\prime} B_{k}^{\prime}-\left(A_{k}^{\prime}-B_{k}^{\prime}\right)^{2}\right|_{p}=\left|y^{2}-\left(A_{k}^{\prime}+B_{k}^{\prime}\right)^{2}\right|_{p} \leq p^{-\lambda}
$$

and, by (30) and $\left|A_{k}^{\prime}-B_{k}^{\prime}\right|_{p}^{2} \geq p^{-\lambda+1}$, we get

$$
\left|\frac{y^{2}-4 A_{k}^{\prime} B_{k}^{\prime}}{\left(A_{k}^{\prime}-B_{k}^{\prime}\right)^{2}}-1\right|_{p} \leq p^{-1} .
$$

Moreover the number $\left(y^{2}-4 A_{k}^{\prime} B_{k}^{\prime}\right) /\left(A_{k}^{\prime}-B_{k}^{\prime}\right)^{2}$ lies in $\mathbb{Q}_{p}$, since in the case where $\omega \notin \mathbb{Q}_{p}$, it is invariant under the above automorphism $\sigma$. Inequality (36) allows us to define the number $\sqrt{\left(y^{2}-4 A_{k}^{\prime} B_{k}^{\prime}\right) /\left(A_{k}^{\prime}-B_{k}^{\prime}\right)^{2}}$ in $\mathbb{Q}_{p}$, and we put in (33)

$$
\sqrt{y^{2}-4 A_{k}^{\prime} B_{k}^{\prime}}=\left(A_{k}^{\prime}-B_{k}^{\prime}\right) \sqrt{\frac{y^{2}-4 A_{k}^{\prime} B_{k}^{\prime}}{\left(A_{k}^{\prime}-B_{k}^{\prime}\right)^{2}}}
$$

Thus $x$ (see (33)) is well defined in $\mathbb{Q}_{p}(\omega)$. Further, let us prove that

$$
|x-1|_{p} \leq|\omega-1|_{p} .
$$

Indeed, writing

$$
\left|\sqrt{y^{2}-4 A_{k}^{\prime} B_{k}^{\prime}}-\left(A_{k}^{\prime}-B_{k}^{\prime}\right)\right|_{p}=\left|A_{k}^{\prime}-B_{k}^{\prime}\right|_{p} \cdot\left|\sqrt{\frac{y^{2}-4 A_{k}^{\prime} B_{k}^{\prime}}{\left(A_{k}^{\prime}-B_{k}^{\prime}\right)^{2}}}-1\right|_{p}
$$

we deduce from (34) that

$$
\left|\sqrt{y^{2}-4 A_{k}^{\prime} B_{k}^{\prime}}-\left(A_{k}^{\prime}-B_{k}^{\prime}\right)\right|_{p}=\frac{\left|y^{2}-4 A_{k}^{\prime} B_{k}^{\prime}-\left(A_{k}^{\prime}-B_{k}^{\prime}\right)^{2}\right|_{p}}{\left|A_{k}^{\prime}-B_{k}^{\prime}\right|_{p}},
$$


and from (35) that

$$
\left|\sqrt{y^{2}-4 A_{k}^{\prime} B_{k}^{\prime}}-\left(A_{k}^{\prime}-B_{k}^{\prime}\right)\right|_{p} \leq \frac{p^{-\lambda}}{\left|A_{k}^{\prime}-B_{k}^{\prime}\right|_{p}} .
$$

Since $\left|A_{k}^{\prime}-B_{k}^{\prime}\right|_{p} \geq p^{-\lambda / 2}$, it follows that

$$
\left|\sqrt{y^{2}-4 A_{k}^{\prime} B_{k}^{\prime}}-\left(A_{k}^{\prime}-B_{k}^{\prime}\right)\right|_{p} \leq p^{-\lambda / 2}
$$

As $\left|y-\left(A_{k}^{\prime}+B_{k}^{\prime}\right)\right|_{p} \leq p^{-\lambda}$, we thus get

$$
\left|y+\sqrt{y^{2}-4 A_{k}^{\prime} B_{k}^{\prime}}-2 A_{k}^{\prime}\right|_{p} \leq p^{-\lambda / 2},
$$

which leads to $(37)$ since $\left|A_{k}^{\prime}\right|_{p} \geq 1$.

Lastly we prove that $\log x / \log \omega$ belongs to $\mathbb{Q}_{p}$. Indeed, in the case where $\omega \notin \mathbb{Q}_{p}$, we have

$$
\sigma\left(\sqrt{\frac{y^{2}-4 A_{k}^{\prime} B_{k}^{\prime}}{\left(A_{k}^{\prime}-B_{k}^{\prime}\right)^{2}}}\right)=\sqrt{\frac{y^{2}-4 A_{k}^{\prime} B_{k}^{\prime}}{\left(A_{k}^{\prime}-B_{k}^{\prime}\right)^{2}}}
$$

since this number lies in $\mathbb{Q}_{p}$. Hence, as $\sigma\left(A_{k}^{\prime}-B_{k}^{\prime}\right)=B_{k}^{\prime}-A_{k}^{\prime}$, we get $\sigma\left(\sqrt{y^{2}-4 A_{k}^{\prime} B_{k}^{\prime}}\right)=-\sqrt{y^{2}-4 A_{k}^{\prime} B_{k}^{\prime}}$. We thus have

$$
\sigma\left(\frac{y+\sqrt{y^{2}-4 A_{k}^{\prime} B_{k}^{\prime}}}{2 A_{k}^{\prime}}\right)=\frac{y-\sqrt{y^{2}-4 A_{k}^{\prime} B_{k}^{\prime}}}{2 B_{k}^{\prime}} .
$$

It follows immediately that

$$
\sigma(x)=1 / x
$$

and as $\omega$ also satisfies (38), we conclude as above that $\log x / \log \omega$ belongs to $\mathbb{Q}_{p}$. Moreover, as $|\log x / \log \omega|_{p}=|x-1|_{p} /|\omega-1|_{p}$, we conclude from (37) that $\log x / \log \omega$ belongs to $\mathbb{Z}_{p}$. Accordingly, given a positive integer $N$, there exists an integer $n$, with $0 \leq n<p^{N}$, such that

$$
\left|\frac{\log x}{\log \omega}-n\right|_{p} \leq p^{-N}
$$

i.e., $\left|\log x-\log \omega^{n}\right|_{p} \leq p^{-N}|\omega-1|_{p}$, and thus $\left|x-\omega^{n}\right|_{p} \leq p^{-N}|\omega-1|_{p}$. As $\left|B_{k}^{\prime}\right| \leq\left|A_{k}^{\prime}\right| \leq 1 /|\omega-1|_{p}$, we thus get

$$
\left|\varphi_{k}(x)-\varphi_{k}\left(\omega^{n}\right)\right|_{p} \leq p^{-N}
$$

Now $\varphi_{k}(x)=y$, and if $0 \leq n \leq h_{k}$, then $\varphi_{k}\left(\omega^{n}\right)=L_{k} q_{m_{k}+n T}$. Let us select $k$ such that $h_{k} \geq p^{N}$; we have thus found an integer $n$, with $0 \leq n \leq h_{k}$, such that

$$
\left|L_{k} q_{m_{k}+n T}-y\right| \leq p^{-N}
$$

Accordingly, the $p$-adic closure of the set of $L_{k} q_{m_{k}+n T}$ with $k \geq 1$ and $0 \leq n \leq h_{k}$ contains the ball $|y-1|_{p} \leq p^{-\lambda}$. 
There are some minor changes when $p=2$. First note that, using the logarithm function over $\mathbb{Q}_{2}$ for $|z-1| \leq 1 / 4$, we get $\left|z^{2}-1\right|_{2}=\frac{1}{2}|z-1|_{2}$. The function $\sqrt{\cdot}$ is then defined over the ball $|z-1|_{2} \leq 1 / 8$ in $\mathbb{Q}_{2}$, and satisfies $|\sqrt{z}-1|_{2}=2|z-1|_{2}$. Also note that if $\left|A_{k}-B_{k}\right|_{2}<1$, then $\left|A_{k}\right|_{2}=\left|B_{k}\right|_{2}=2$, since $\left|A_{k}+B_{k}\right|_{2}=1$. Reasoning as above, we may thus suppose that

$$
\left|A_{k}-B_{k}\right|_{2} \geq \frac{1}{2}|\omega-1|_{2} .
$$

The number $\lambda$ is then determined by

$$
2^{-\lambda}=\frac{1}{16}|\omega-1|_{2}^{2}
$$

and $L_{k}$ is determined by

$$
L_{k} q_{m_{k}} \equiv 1 \bmod 2^{\lambda}
$$

and $0<L_{k}<2^{\lambda}$. Then the 2 -adic closure of the set of integers $L_{k} q_{m_{k}+n T}$ contains the ball $\left\{y:|y-1|_{2} \leq 2^{-\lambda}\right\}$, and (39) holds.

In order to obtain (7), it is enough to note that $\log \left|L_{k} q_{m_{k}+n T}\right| \ll m_{k}+n$, and thus, if condition (6) is satisfied, then

$$
\log \left|L_{k} q_{m_{k}+n T}\right| \ll h_{k} \text {. }
$$

We may choose the integer $N$ above such that $h_{k} / p<p^{N} \leq h_{k}$, i.e., $p^{N} \asymp h_{k}$, and from (39), we get (7).

4. Proof of Theorem 3. Let $\alpha$ be as in the statement of Theorem 3 . Let $p$ be a prime number. First, notice that if $\omega$ is any quadratic unit, then the index of the multiplicative group $G=\left\{x \in \mathbb{Q}_{p}(\omega):|x-1|_{p}<p^{-1 /(p-1)}\right\}$ in the unit ball $\left\{x \in \mathbb{Q}_{p}(\omega):|x-1|_{p}=1\right\}$ is a divisor of $p^{2}\left(p^{2}-1\right)$. Hence, $\left|\omega^{p^{2}\left(p^{2}-1\right)}-1\right|_{p}<p^{-1 /(p-1)}$. Accordingly, in the statement of Theorem 3, if we replace $T$ by $p^{2}\left(p^{2}-1\right) T$, we can suppose, as in the proof of Theorem 1 , that the eigenvalues $\omega_{T}, 1 / \omega_{T}$ of the matrix $M=M_{T} \ldots M_{1}$ are quadratic units with $\left|\omega_{T}^{p^{2}\left(p^{2}-1\right)}-1\right|_{p}<p^{-1 /(p-1)}$.

Thus, in order to prove Theorem 3 for $y=0$ it is enough to consider $Q_{n}=q_{n T-1}$ for $0 \leq n \leq h$. This sequence satisfies (10), hence we can write

$$
Q_{n}=A\left(\omega_{T}^{n}-\omega_{T}^{-n}\right)
$$

since $Q_{0}=0$. We have

$$
|A|_{p} \leq 1 /\left|\omega_{T}^{2}-1\right|_{p}
$$

Hence, for $0<s \leq \log h / \log p$, we have

$$
\left|Q_{p^{s}}\right|_{p} \leq \frac{\left|\omega_{T}^{2 p^{s}}-1\right|_{p}}{\left|\omega_{T}^{2}-1\right|_{p}}=p^{-s} .
$$

Taking $s$ such that $h / p<p^{s} \leq h$, we get $\left|Q_{p^{s}}\right|_{p} \ll 1 / h$. This proves the result, in view of $(28)$. 
Let $y$ be a non-zero integer. Consider $Q_{n}^{\prime}=y q_{n T}=E \omega_{T}^{n}+F \omega_{T}^{-n}$, where

$$
\max \left\{|E|_{p},|F|_{p}\right\} \leq 1 /\left|\omega_{T}^{2}-1\right|_{p} .
$$

Since $Q_{0}^{\prime}=y$, we have as above

$$
\left|Q_{2 p^{s}}^{\prime}-y\right|_{p} \leq \frac{\left|\omega_{T}^{2 p^{s}}-1\right|_{p}}{\left|\omega_{T}^{2}-1\right|_{p}}=p^{-s},
$$

which again proves the result by (28).

Remarks on the proofs. Although Theorem 2 for $y=0$ is a consequence of Theorem 1, and was already proved in [12], we would like to note that in this case, the proof of (7) is very simple, and the use of the $p$-adic logarithm function is actually not necessary. Indeed, the assumptions of Theorem 1 are satisfied for some positive integer $m$, and we have $a_{m+j+n T}=b_{j}$ for every non-negative integer $n$ and for any $j=1, \ldots, T$. For any $k \geq 1$, we set $m_{k}=m$, and we choose the integers $x_{k}=q_{m+T}$ and $y_{k}=-q_{m}$. Then, as in the proof of Theorem 1, we consider the integers $Q_{n}=q_{m+T} q_{m+n T}-$ $q_{m} q_{m+(n+1) T}$. They satisfy (28) since $m$ is now a fixed number. In this case, as $Q_{0}=0$, we have $C_{k}=-D_{k}$, that is to say, we can write $Q_{n}=C\left(\omega^{n}-\omega^{-n}\right)$ with $C \neq 0$ since $q_{m+T}-\omega q_{m} \neq 0$. Hence, $Q_{n} \neq 0$ for $n>0$, and

$$
\left|Q_{n}\right|_{p} \ll\left|\omega^{2 n}-1\right|_{p}
$$

Therefore, we only have to check that

$$
\left|\omega^{2 p^{s}}-1\right|_{p} \leq p^{-s}\left|\omega^{2}-1\right|_{p}
$$

This follows from an elementary induction by writing $\omega^{2 p^{s}}=1+u_{s}$ and using Newton's formula. As $\log Q_{n} \ll n$, we thus see that the integers $Q_{p^{s}}$ satisfy (28) with $\log \left|Q_{p^{s}}\right| \ll p^{s}$ and $\left|Q_{p^{s}}\right|_{p} \ll p^{-s}$. This provides the estimate (7). The same holds when $y \neq 0$ is an integer. Indeed, by the Bézout theorem, $q_{m}$ and $q_{m+1}$ being coprime, we can take integers $x^{\prime}$ and $y^{\prime}$ such that $x^{\prime} q_{m}+$ $y^{\prime} q_{m+1}=y$ (where $m \geq 0$ is chosen in a such way that $a_{m+j+n T}=b_{j}$ for every non-negative integer $n$ and for any $j=1, \ldots, T)$. Since both the sequences $\left(q_{m+n T}\right)_{n \geq 0}$ and $\left(q_{m+1+n T}\right)_{n \geq 0}$ satisfy (10), for every non-negative integer $n$ we can write

$$
x^{\prime} q_{m+n T}+y^{\prime} q_{m+1+n T}=E \omega^{n}+F \omega^{-n}
$$

where $E$ and $F$ are in $\mathbb{Q}_{p}(\omega)$ with $|E|_{p} \leq 1 /\left|\omega^{2}-1\right|_{p}$ and $|F|_{p} \leq 1 /\left|\omega^{2}-1\right|_{p}$. The integers $Q_{n}^{\prime}=x^{\prime} q_{m+n T}+y^{\prime} q_{m+1+n T}$ satisfy (28). Since $\left|\omega^{2 p^{s}}-1\right|_{p} \leq$ $p^{-s}\left|\omega^{2}-1\right|_{p}$, we see that for $n=2 p^{s}$ we have $\left|Q_{2 p^{s}}^{\prime}-Q_{0}^{\prime}\right|_{p} \leq p^{-s}$, that is to say,

$$
\left|Q_{2 p^{s}}^{\prime}-y\right|_{p} \leq p^{-s} \text {. }
$$

Further, it is easy to see that Theorems 2 and 3 remain valid upon replacing in (8) the $p$-adic absolute value $|\cdot|_{p}$ by $|\cdot|_{\mathcal{D}}$, if we take $y \in \mathbb{Z}$. 
In the other parts of the proof, the role played by the $p$-adic logarithm function seems deeper. For instance, it is easy to prove the analogue of Theorem 2 for formal power series over a finite field, when $y$ is a polynomial. But we do not know whether Theorem 1 has an analogue in this setting. The crucial point is the use of the $p$-adic logarithm function, which we lose in the formal power series case.

5. Proof of Theorem 4. Let $p$ be a prime number and $y$ be an integer. Let $\mu$ denote the Kaufman measure, whose existence has been proved in [7]. The measure $\mu$ is supported on the set $\boldsymbol{B a d}$. For any $n \geq 1$, set $q_{n}:=p^{n}+y$. For any real number $\alpha$ we have

$$
\left|q_{n}\right| \cdot\left\|q_{n} \alpha\right\| \cdot\left|q_{n}-y\right|_{p}=\left|q_{n}\right| \cdot\left\|q_{n} \alpha\right\| \cdot\left|p^{n}\right|_{p} \leq(1+|y|)\left\|q_{n} \alpha\right\| .
$$

Thus, (4) holds as soon as $\alpha$ satisfies

$$
\inf _{n \geq 1}\left\|q_{n} \alpha\right\|=0 .
$$

Using the exponential growth of the sequence $\left(q_{n}\right)_{n \geq 1}$ and a result of Davenport, Erdős, and LeVeque [4], as explained in Section 4 of [13], we infer that (40) holds for $\mu$-almost all $\alpha$. Hence, $\mu$-almost all $\alpha$ satisfy (4) for any prime $p$ and any integer $y$. Arguing then as on p. 294 of [13], we conclude that the Hausdorff dimension of the set of real numbers $\alpha$ in $\boldsymbol{B a d}$ for which (4) holds for any prime $p$ and any integer $y$ is equal to 1 , as claimed.

6. Proof of Theorem 5. Our proof is very much inspired by [8]. It rests on the folding lemma, recalled below.

Lemma F. If $p_{n} / q_{n}=\left[a_{0} ; a_{1}, a_{2}, \ldots, a_{n}\right]$ with $a_{n} \geq 2$, then

$$
\frac{p_{n}}{q_{n}}+\frac{(-1)^{n}}{q_{n}^{2}}=\left[a_{0} ; a_{1}, a_{2}, \ldots, a_{n-1}, a_{n}+1, a_{n}-1, a_{n-1}, \ldots, a_{2}, a_{1}\right] .
$$

Lemma F follows from Propositions 2 and 3 of [14].

Recall that $\left[0 ; a_{1}, \ldots, a_{n}, 1,1\right]=\left[0 ; a_{1}, \ldots, a_{n}, 2\right]$ for any positive integers $a_{1}, \ldots, a_{n}$. We display an immediate consequence of Lemma $\mathrm{F}$. then

Lemma 3. If $a / m=\left[0 ; 1,1, a_{3}, \ldots, a_{h-1}, a_{h}\right]$ with $h \geq 4$ and $a_{h} \geq 2$,

$$
\frac{m a+(-1)^{h}}{m^{2}}=\left[0 ; 1,1, a_{3}, \ldots, a_{h-1}, a_{h}+1, a_{h}-1, a_{h-1}, \ldots, a_{3}, 2\right] .
$$

Lemma 3 is the main tool for the proof of Theorem 5 . Let $p$ be a prime number. Observe that in the open real interval with endpoints $[0 ; 1,1,1,2]$ and $[0 ; 1,1,1,3]$ there are rational numbers whose denominator is a power of $p$. Consequently, there exist positive integers $a$ and $b$, with $1 \leq a \leq p^{b}$ 
and $a$ coprime with $p$, such that

$$
\frac{a}{p^{b}}=\left[0 ; 1,1, a_{3}, \ldots, a_{h-1}, a_{h}\right]
$$

with $h \geq 4$ and $a_{h} \geq 2$. Set

$$
M=\max \left\{a_{3}, \ldots, a_{h-1}, a_{h}+1\right\} .
$$

By repeated application of Lemma 3 , we see that, for any $j \geq 2$, the continued fraction of the rational number

$$
\alpha_{j}:=\frac{a}{p^{b}}+\frac{(-1)^{h}}{p^{2 b}}-\frac{1}{p^{2^{2} b}}-\cdots-\frac{1}{p^{2^{j} b}}
$$

reads $[0 ; 1,1, \ldots, 2]$ and has all its partial quotients bounded by $M$.

Set

$$
\alpha=\lim _{j \rightarrow \infty} \alpha_{j}=\frac{a}{p^{b}}+\frac{(-1)^{h}}{p^{2 b}}-\sum_{j \geq 2} \frac{1}{p^{2^{j b}}} .
$$

By construction, all the partial quotients of $\alpha$ are less than or equal to $M$, hence $\alpha$ is in $\boldsymbol{B a d}$. Furthermore, it is easily checked that, for any $j \geq 2$, we have

$$
p^{2^{j} b} \cdot\left\|p^{2^{j} b} \alpha\right\| \leq 1+2 p^{-2^{j+1} b} .
$$

This implies that

$$
\liminf _{q \rightarrow \infty} q^{2} \cdot\|q \alpha\| \cdot|q|_{p} \leq 1
$$

as asserted.

\section{References}

[1] B. Adamczewski and Y. Bugeaud, On the Littlewood conjecture in simultaneous Diophantine approximation, J. London Math. Soc. 73 (2006), 355-366.

[2] - - - On the Maillet-Baker continued fractions, J. Reine Angew. Math., to appear.

[3] J. W. S. Cassels and H. P. F. Swinnerton-Dyer, On the product of three homogeneous linear forms and indefinite ternary quadratic forms, Philos. Trans. Roy. Soc. London Ser. A 248 (1955), 73-96.

[4] H. Davenport, P. Erdős and W. J. LeVeque, On Weyl's criterion for uniform distribution, Michigan Math. J. 10 (1963), 311-314.

[5] M. Einsiedler, A. Katok and E. Lindenstrauss, Invariant measures and the set of exceptions to the Littlewood conjecture, Ann. of Math. 164 (2006), 513-560.

[6] M. Einsiedler and D. Kleinbock, Measure rigidity and p-adic Littlewood-type problems, Compos. Math. 143 (2007), 689-702.

[7] R. Kaufman, Continued fractions and Fourier transforms, Mathematika 27 (1980), $262-267$.

[8] T. Komatsu, On a Zaremba's conjecture for powers, Sarajevo J. Math. 1 (2005), 9-13.

[9] J. E. Littlewood, Some Problems in Real and Complex analysis, D. C. Heath and Raytheon Education Co., Lexington, MA, 1968. 
[10] B. de Mathan, Conjecture de Littlewood et récurrences linéaires, J. Théor. Nombres Bordeaux 13 (2003), 249-266.

[11] -, On a mixed Littlewood conjecture for quadratic numbers, ibid. 17 (2005), 207215.

[12] B. de Mathan et O. Teulié, Problèmes diophantiens simultanés, Monatsh. Math. 143 (2004), 229-245.

[13] A. D. Pollington and S. Velani, On a problem in simultaneous Diophantine approximation: Littlewood's conjecture, Acta Math. 185 (2000), 287-306.

[14] A. J. van der Poorten and J. Shallit, Folded continued fractions, J. Number Theory 40 (1992), 237-250.

[15] H. P. Schlickewei, The p-adic Thue-Siegel-Roth-Schmidt theorem, Arch. Math. (Basel) 29 (1977), 267-270.

Mathématiques

Université Louis Pasteur

7 , rue René Descartes

67084 Strasbourg Cedex, France

E-mail: bugeaud@math.u-strasbg.fr

Institut de Mathématiques

Université Bordeaux I

351, cours de la Libération

33405 Talence Cedex, France

E-mail: demathan@math.u-bordeaux1.fr
Institut für Diskrete Mathematik und Geometrie TU Wien A-1040 Wien, Austria E-mail: michael.drmota@tuwien.ac.at

Received on 31.5.2006

and in revised form on 13.3.2007 